\title{
Enabling Knowledge Broker Analysis through Actor Clusters in Organizational Structures in Enterprise Social Media
}

\author{
Mia Leppälä \\ Aalto University \\ $\underline{\text { mia.leppala@aalto.fi }}$
}

\author{
Jukka Huhtamäki \\ Tampere University \\ jukka.huhtamaki@tuni.fi
}

\begin{abstract}
Knowledge brokers serve as facilitators of knowledge sharing. The extant literature calls for nuanced analyses of different organizational structures as the spaces knowledge brokers operate in. Our interest lies in formal, semiformal, and informal organizational network structures and in how knowledge brokers are positioned in them. In this paper, we outline a collaborative analysis method, with researchers from different disciplines working together in data sprints. The benefit of this process is that it enables analyzing large organizational networks with deep insights. Amplifying social network analysis with field knowledge offers a deeper understanding of the connections in the network. This paper describes the analysis process and proposes interdisciplinary data processing techniques. We applied the proposed method using an extensive empirical data set that includes intraorganizational social media interactions between employees in a global organization. Our analysis transforms enterprise social media data into a network model that describes an organization's social structure.
\end{abstract}

\section{Introduction}

Remote work and global teams are already commonplace, and the COVID-19 pandemic has significantly reinforced the trend [1,2]. The increasing use of technology affects the way in which people organize and collaborate in organizations [3,4]. New technologies facilitate knowledge sharing and online collaboration and enable distributed work settings in which knowledge brokers play an important role in connecting other people and issues, thus acting as facilitators of knowledge sharing [5].

Prior studies on knowledge brokering have emphasized the role of knowledge brokers as bridges between research and practice or as translators of specialized knowledge or as collaboration facilitators
[5,6,7]. A network perspective on knowledge brokers highlights their role in bridging gaps between individuals, groups or clusters, also known as structural holes [8]. Organizational network structures are important because they affect both the work processes and the flow of knowledge throughout an organization. We add an individual actor cluster perspective to the discussion and investigate the structural positions of knowledge brokers in different organizational structures: formal, semiformal, and informal $[9,10]$. Various groups are important when forming the network representation of an organization. Our analysis reconstructs the organizational structure based on the connections in the network and includes different types of groups according to their formality. New ways of working, such as the use of enterprise social media (ESM), and an agile culture are shifting traditional organizational structures from formal to semiformal and informal. Formal organizational structure refers to official rules and practices and goals to be achieved through certain work processes [9]. Informal, on the other hand, refers to a structure based on informal relationships that might arise, for example, from coffee table discussions between colleagues on the same or different hierarchical levels or from different departments [9]. In semiformal organizational structures, which lie between the formal and informal structures, official work structures are reinforced by emerging informal relationships between employees [10].

We believe that ESM reduces the importance of traditional organizational structures and enables the members of an organization to act more freely [11]. By providing the affordances of visibility and association, ESM offers employees opportunities to form groups based on mutual interests rather than hierarchies, rules, and authority. Interactions on ESM are visible not only to senders and receivers but also to a large network of other people who can see the messages and connections between the senders and receivers. This gives knowledge brokers the opportunity to obtain information that they deem worth transferring. As 
Leonardi [12] notes, ESM serves as a tool for making communication visible, thus promoting the development of communal knowledge of who knows whom and what. ESM provides the means to form dense informal connections between organizational actors. Informal structures are important and relevant because they allow people, ideas, and knowledge to flow within the organization, thus creating a space for informal encounters [10].

Using a network analysis lens to study organizational structures introduces the actor cluster perspective, according to which clusters are formed by the interactions in the network. These clusters are formal, semiformal, or informal, depending on how they have been formed. We developed a method to support the network analysis based on the interactions in the network.

To investigate knowledge brokering, the approach developed in this study is based on the use of digital traces accumulating on ESM to construct an organizational social network. Following Williams and Shepherd [13], we combined the expertise of a data scientist with that of a qualitative researcher to gain a deeper understanding of the network structure and the connections and structural positions of knowledge brokers in the network. Intensive data sprints are becoming increasingly common, for example, in digital ethnography, to facilitate interdisciplinary collaboration in thorough investigations that combine computational and qualitative approaches [14,15]. The proposed approach uses social network analysis [16] and visual network analysis $[17,18]$ to identify the structure of an organizational network, the structural positions of individual actors, and the emerging actor clusters in the network. The study aimed to answer the following research question: "How can organizational social structures be modeled (using ESM data) to support knowledge brokering analysis?"

\section{Research design}

\subsection{Context}

The context of our study is ESM, which refers to technologies used to discuss, coordinate, and collaborate in organizations [19,20]. These platforms allow discussions to be visible throughout the organizational social network and provide a space for knowledge brokers to share knowledge. Research benefits from ESM platforms, as they provide easy access to interaction data that would otherwise be difficult to obtain. The affordances of ESM include visibility, association, editability, and persistence [21]. Visibility allows more people to see what is going on in the organization, while association enables linking practices, thus improving knowledge sharing. For example, when messages are available to everyone, active collaborators can learn who knows what, which enables them to connect people with mutual needs. In our study, we consider knowledge brokers as a type of actor who links people in the network. Knowledge brokers are traditionally seen as bridges, translators, and facilitators in knowledge networks [6,7]. The other two affordances, editability and persistence, provide tools for correcting misinformation and, as messages are continuously accessible, support asynchronous communication.

\subsection{Empirical case and data}

Our extensive empirical data allows us to conduct a longitudinal study of a global organization. The case organization is a consumer electronics and telecommunications hardware manufacturer that employs over 30,000 people worldwide. Its ESM plays an important role in communication between organizational members, ranging from ICT, R\&D, and marketing to strategy discussions.

We focused on written messages, which are important for knowledge sharing, as the organizational members are dislocated. Messages are sent to individuals or groups via an internal ESM platform, and the sender can choose to whom a message is sent. Everyone in the organization can use the platform and see the messages if they are not restricted to a specified group or person. The ESM is constructed based on connections that represent messages exchanged between users. The message that opens a discussion includes the name of the sender and the recipient, who can be one or more individuals or groups. The sender attributes contain the name of the individual sending the message, the name of the group, if the sender has selected any, and the time when the message is sent. Commenting messages are always directed at the sender of the opening message. Each comment has the name of the sender and the time when the comment is sent. The message thread is built under the sender. Thus, each comment can be seen below the opening message.

Our data set contains the communication of knowledge workers who used computers for their daily work. There were 35,900 registered users in the ESM, which also included people whose work did not involve knowledge sharing, such as assembly line workers. The data set includes 9,000 employees who contributed to the ESM platform at least once. The conversations between these employees encompassed a wide variety of topics, mostly related to work, over almost four years. Overall, the data set consists of 32,902 message threads and 124,015 messages. According to the guidelines of 
the Finnish National Board on Research Integrity, this study did not require institutional ethical approval [22].

\subsection{Method}

We performed a social network analysis $[13,16,18]$ to identify the structural positions of individual actors in the organizational social structure as they emerge from the ESM and to find clusters of actors in the interaction network. To manage the network construction and analysis process, we applied the Ostinato model [17], which defines an iterative and incremental process for transforming source data into a network and analyzing the resulting network through interactive visualization.

We worked together in data sprints [14,15], intensive collaboration sessions, in which the analytical process was implemented and revised in an iterative and incremental manner. Data sprints facilitated learning within the investigative team: the researcher with experience in qualitative analysis was able to learn about data processing, and the data scientist gained knowledge of brokering as a theoretical concept. Most importantly, detailed decisions on data preprocessing, filtering, and transforming and network construction were made collaboratively. Data sprints usually refer to intensive research and coding workshops in which interdisciplinary research groups work together physically [15]. Due to the COVID-19 pandemic, we were unable to meet in person and therefore collaborated in real time via Zoom.

Following Freeman [18], we first identified patterns emerging in the social network and made sense of the network by sharing them with others-in this case, between the authors. We performed the analysis in Python in a Jupyter Notebook environment. Jupyter Notebooks are analytical notebooks that combine textual documentation of the analysis process with Python code that implements the process and output of the code, including data aggregates, listings, visualizations, and other representations, following the principles of literate programming [23]. Contextual documentation allowed the investigative team to incrementally build a shared understanding of the process. Data aggregates, listing, visualizations, and other forms of analysis process output facilitate data exploration and description, which are a necessary part of analyses that combine qualitative and computational approaches. The notebooks serve as boundary objects [24] that facilitate data-intensive analyses of organizational social structures.

\subsection{Network modeling rules and principles}

Every network model is composed of nodes and edges. In constructing the network model of the interactions on the ESM under investigation, we created nodes for each user, group, and business unit appearing in the source data. Business units represented the formal component of the organizational structure, interactions between users constituted the informal component, and the ESM groups represented the semiformal component. The ESM groups were teams, projects, and other communities with mutual interests that were formed on the ESM platform by employees.

We formed edges between nodes according to a set of rules. First, for each user sending an opening message, we connected the discussion to each group and user to whom the message was sent. Second, for each comment, we created a network node for the commenter and connected it to the message sender node. The sender was the person starting the message thread. Third, we created a node each time a group or user was mentioned in a message or comment and formed a connection to the node from the mentioning user node. All the connections were directed, pointing from the user initiating the interaction. Connection weights represented the number of interactions between a pair of nodes.

In actor-level social network analysis, we focused on three main measures: weighted indegree, weighted outdegree, and betweenness centrality [25]. At the network level, we applied cluster detection to identify actor clusters [26]. We chose these measures because we were interested in bridge users, called knowledge brokers, and communities that could be identified in the network structure. Weighted indegree identifies the actors that attract the most attention in the network. These actors receive the most messages or are the most frequently mentioned by others. Weighted outdegree, on the other hand, highlights the actors who send the most messages and can be regarded as the most actively forming connections in the network. Betweenness centrality is important when describing which nodes are the most central between other nodes. Thus, in our analysis, high betweenness centrality indicated individuals who occupied central positions, linking actor clusters.

\subsection{Data preprocessing}

In this section, we describe the analysis process in detail to provide an example of the multiple phases required and highlight the significance of each decision in the analysis.

Following the Ostinato model [17], the analysis process started with collecting and aggregating source data. We obtained the data in two files: a data dump exported from the ESM in XML format, including all the messages and comments sent during the study period, and a spreadsheet containing the user details. 
First, we processed the XML file to extract the messages and comments and their metadata, including the actors and groups related to each message and the time of each message. The users and groups mentioned in messages and comments needed to be explicitly parsed from message content using @ mentions. Second, we merged additional user attributes from the spreadsheet with the message and comment data using ESM IDs and usernames as unique identifiers. There were two kinds of message senders: individuals and groups.

While constructing the network, we considered all the connections between senders and receivers, as well as mentions, which are important for identifying key individuals in the network. When a person is mentioned by others, this usually indicates that this individual may know something related to the message. In the beginning, we extracted the users from the messages and comments. Then, we extracted all the groups and explored the statistics related to each group. Thus, we were able to see the number of messages posted to each group.

We built a network for user data from which we extracted details about individual actors, thus creating an actor network. We identified the connections between actors, including connections between individual actors and groups and added the actor details to the network.

In our data, @ mentions could refer to users, groups, or even actors who did not have an ID in ESM, as they had not yet been registered. We also realized that $@$ mentioned users were included in the data by their names, not by their IDs. Thus, we needed to make a list of actors by their names and match them to their IDs, which we extracted from the original actor spreadsheet.

After identifying individual users and groups in the messages and the@mentions and business units, we formed a network of all the actors. We checked the network nodes once again. Here, we had again a decision to make, as we noticed errors when extracting all the IDs: some IDs were not linked to any of the defined actors (users, groups, or business units). Thus, we had two kinds of actors: those with numerical IDs and those with names (the business units). To be consistent, we could have created IDs for business units as well.

After these construction steps, we had a network that could be inspected to see if it looked the same as before these new revisions to the data processing pipeline, both visually and using node counts and other metrics. Verifying became easier because one of the authors had knowledge of where the different names came from - which were business units and which were groups, for example.
When focusing on groups, we found an additional error, as some of them did not have names. We discussed various causes and resolved the issue. This open discussion between the authors during the data sprints was crucial for identifying errors and arriving at solutions. The authors collaboratively found ways to correct the errors or, if not possible, decided how to proceed.

\subsection{Network visualization}

Once the network model representing the interactions on the ESM was formed, we exported it in GEXF, a network-specific markup language, and imported the data into Gephi, an open-source network exploration, analysis, and visualization platform [27]. To visualize the network, we first calculated the network metrics for each actor in the network. We measured the weighted indegree, weighted outdegree, and betweenness centrality (using both directed and undirected variants) to identify actor clusters.

Visual analysis of a network is based on an iterative process whereby the visual properties of the nodes and edges and the entire network are determined using source data and network metrics. In the first step of the visual analysis, we used a force-directed layout algorithm, Force Atlas 2, to determine the position of each network node in a way that best allowed us to observe the emerging structural patterns in the network. Next, we used colors to represent each node's network cluster membership. While maintaining the node positions, we created a separate representation of the network for each network metric to allow the analysis of the structural position of each actor. To further support the interactive analysis, we visualized the different network representations with GEXF.js (https://github.com/raphv/gexf-js), a JavaScript-based network exploration tool.

\section{Case vignette: Knowledge brokering in organizational network structures}

Once we constructed the network representation of the ESM, we proceeded to explore it to gain insight into the structural positions of knowledge brokers. To make sense of knowledge brokering in the context of formal, semiformal, and informal organizational structures, we needed to create several complementary network views, each providing different insights. The creation of these views required the coordinated use of the analytical notebooks and network visualization tools. 


\subsection{Entering the network structure}

The main view of the organizational network structures is presented in Figure 1.

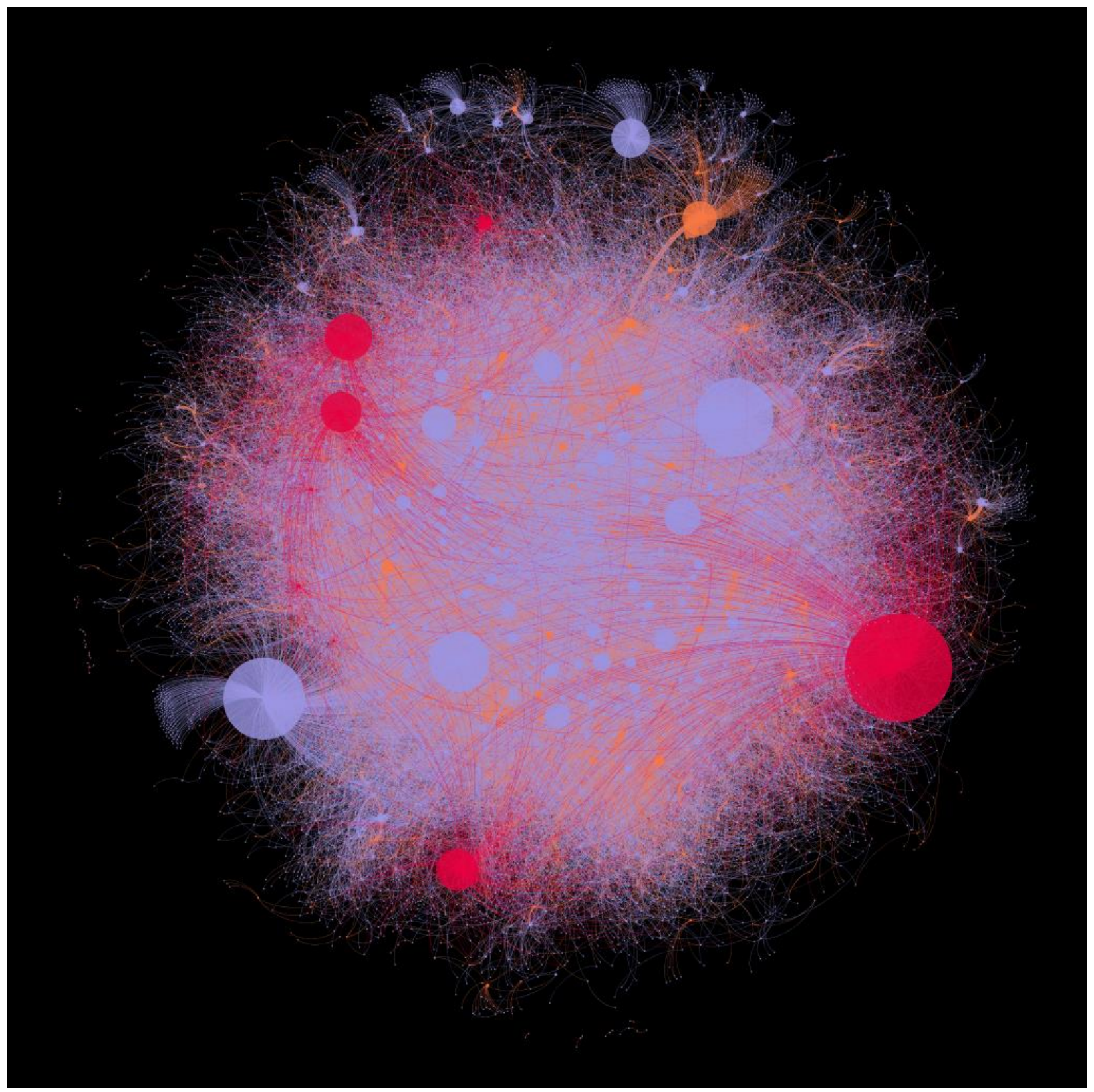

Figure 1. Formal (business units), semiformal (ESM groups), and informal (individuals) network structures. The red nodes represent business units, the orange nodes represent ESM groups, and the purple nodes represent individual users. Betweenness centrality determines the node size.

The main insight gained from Figure 1 is the importance of the different types of nodes in connecting the organization. In this network view, the red nodes represent business units (that is, the formal organizational structure), the orange nodes represent ESM groups (the semiformal structure), and the purple 
nodes represent individual users (the informal structure). The size of each node indicates its betweenness centrality - that is, structural brokerage in connecting the different parts of the network. In network sections where (red) business unit nodes dominate, they serve as the main connecting tissue between the actors. In many cases, this indicates low ESM activity on the part of individual actors. In sections where (orange) group nodes are more prominent, these groups serve as the main connectors, indicating that information and knowledge primarily flow through the groups. Large purple nodes indicate actors who serve as structural brokers, bridging structural holes in the organization. Whether these structural brokers serve as knowledge brokers must be determined by analyzing their actions and the content that they produce on the ESM [5].

\subsection{Focusing on individuals}

An alternative network configuration for exploring the structural positions of individual actors is presented in Figure 2.

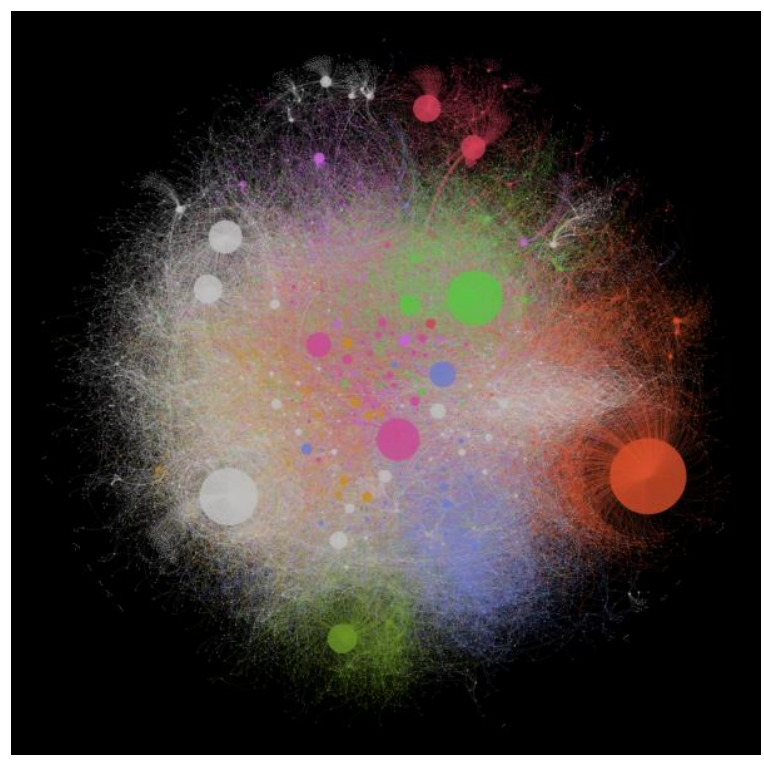

Figure 2. Actor clusters with groups and business units removed. The node colors represent cluster membership. The size of each node is determined by its betweenness centrality, indicating individual actors in a position of structural brokerage.

In this network configuration, each node represents an individual actor - that is, an ESM user. Each node color represents node membership in one of the network clusters. Here, the clusters refer to groups of network nodes that are more connected to each other than the rest of the nodes. The size of each node indicates its betweenness centrality. In this visualization view, we can identify several clusters connected by one or more structural brokers to augment qualitative knowledge broker analysis.

\subsection{Structural brokerage or attention?}

Betweenness centrality can be calculated both by considering the direction of an edge and by allowing the algorithm to traverse edges both ways. In these illustrations, we used the latter approach. To further augment the knowledge broker analysis, Figure 3 presents the network with node size indicating weighted indegree, which serves as a proxy for the attention (number of mentions and responses) that a particular actor receives on the ESM.

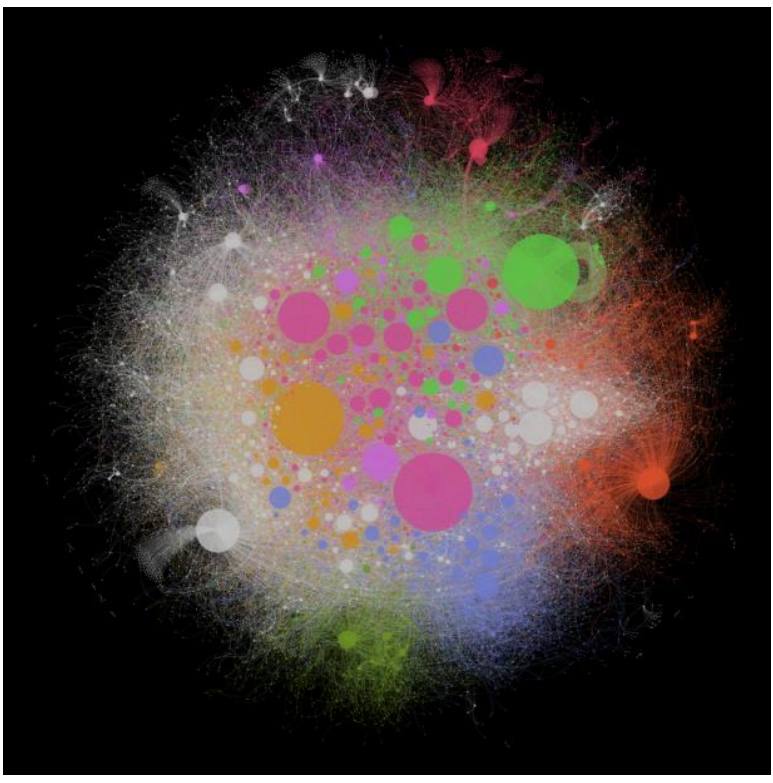

Figure 3. Alternative actor cluster view. The node colors indicate cluster membership. The size of each node is determined by its weighted indegree, which indicates the attention that a particular actor receives.

Static network visualizations are not always the best way to gain insight into the structural positions of individual actors. Therefore, a scatterplot of actors contrasting their indegree or outdegree with their betweenness centrality should complement the analysis. For example, high betweenness centrality and low outdegree would indicate actors with a potential role as knowledge brokers. 


\section{Discussion}

The aim of this study was to design a method to study ESM and knowledge brokering in organizational network structures. We developed a data processing pipeline to construct a network representation of an organization and support knowledge brokering analysis. We defined which actors were presented as nodes and how the nodes were connected to each other. Additionally, we developed principles for network structure analysis and visualization. Using social network analysis and the Ostinato model, we developed different views of the network model. As analysis tools, we chose Jupyter Notebooks and Gephi. To validate our model, we applied it to a case, which we present in this paper. Throughout the analysis process, we collaborated through data sprints on Zoom.

The increased shared understanding that the collaboration afforded enabled us to make better sense of the data and the related data processing methods. Using data sprints in interdisciplinary research enabled the development and modification of our analysis in an iterative and incremental manner. This can be seen as a notebook-centric form of literate computing in which the notebooks serve as objects that allow researchers to cross disciplinary boundaries [24]. For our network modeling, we used nodes to represent the key entities on the ESM-namely, business units representing the formal organizational structure, ESM groups constituting a semiformal structure, and connections between individual actors forming an informal structure. By providing a set of complementary views of the network, we revealed the role of individual actorsthat is, the core of knowledge brokering.

Participants in data sprints usually conduct parts of the work that are time-consuming, such as data cleaning or setting up the infrastructure, beforehand [15]. However, we find that it is important to conduct these processes together since, while cleaning the data, decisions need to be made on what to include and what to exclude. Each decision has a substantial impact on the network structure that emerges from the data through the designed data processing pipeline. Making these decisions is easier if one has prior knowledge of the data, as this advances a shared understanding of both the empirical data and the analytical methods among the investigative team.

Although our data set was overall structured and clean, it required several rounds of cleaning during the analysis process. The data sprints allowed the two investigators to discuss and make shared decisions throughout the cleaning process. Some of the key turning points included the realization that message content included mentioned and tagged users and groups that had to be extracted and their identifiers resolved.
Moreover, we identified messages that were sourced from an external system and therefore lacked sender identifiers. However, as these messages included @ mentions, we needed to include the mentions in these messages to our network. We decided to include these messages only if there was a user ID in the message. By doing so, we added 8,000 new nodes and 20,000 connections to the network. We also realized that the users were able to manually type the @mentioned names, which led to typing errors and inconsistencies in the format of names. To cope with letter case inconsistency, we transformed @mentioned names to lowercase. The message senders and receivers were identified by the original system, so this information was reliable.

Another important decision was related to how to find names with typing errors and match them with the correct names. We omitted these mentions for this analysis. To do this in future studies, we consider building an intelligent system that would identify names in different formats.

Working together in the data sprints made it possible to make these decisions based on each researcher's specific competencies that were relevant to the analysis. This collaborative process enabled a more rigorous analysis in less time.

\section{Conclusion}

A deeper understanding of organizational social structures opens new avenues for research into knowledge brokers as facilitators of knowledge sharing. Methodologically, our study introduces a novel approach to exploring the role of social networks in organizational knowledge transfer and knowledge brokers as a part of it. Working through data sprints allows researchers from different disciplines to collaborate. As the investigators discuss and make common decisions throughout the data process, the analysis is more nuanced and is verified by both qualitative and computational measures.

The proposed network modeling approach allows us to make sense of the formal, semiformal, and informal components of an organization's social structure. ESM mostly includes informal interactionsthat is, interactions that arise from the users' interests and information needs. As such, it is part of the semiformal organizational structure. In the presented case vignette, we used ESM groups as components of the semiformal organizational structure. To include the formal organizational structure in the network representation, we sourced business unit information to complement the view.

We also confirmed the usefulness of betweenness centrality in identifying potential knowledge brokers. 
However, we acknowledge the need to combine a qualitative analysis of message content with a computational analysis of the network structure and the actors' roles in the network to gain deeper insights into knowledge brokers. To facilitate such analyses, we call for new kinds of interactive tools that will allow an interpretation of the network view while qualitatively analyzing message content. Particularly in cases in which the researchers do not have access to the ESM under investigation, we anticipate the need to perform a simplified reconstruction of the original system with message threads, mentions, and user profiles with an augmentation layer that includes computed and qualitatively derived information on actors and content.

Our analysis certainly has limitations. First, the available data on business units were partial. Second, when composing the network representation, we relied on connections that were explicitly available in the system. That is, while we connected users to each other according to responses and tagged mentions, conversations were conducted in message threads without explicitly tagging users. Finally, we did not categorize the individual interactions according to the quality or amount of information exchanged. Therefore, we were unable to filter the network edges according to their function in knowledge exchange.

In future research, we wish to explore network filtering methods for identifying the core social structure of an organization from a knowledge transfer viewpoint, especially the structural holes in it. Moreover, we intend to include computational content analysis to further augment knowledge broker identification and analysis.

\section{References}

[1] Levin D. and T. Kurtzberg, "Sustaining Employee Networks in the Virtual Workplace", MIT Sloan Management Review, Summer 2020 Issue. https://sloanreview.mit.edu/article/sustaining-employeenetworks-in-the-virtual-workplace/amp

[2] Leonardi, P.M., "COVID-19 and the New Technologies of Organizing: Digital Exhaust, Digital Footprints, and Artificial Intelligence in the Wake of Remote Work", Journal of Management Studies, 2020. doi:10.1111/joms.12648

[3] Nordbäck, E.S., K.K. Myers, and R.D. McPhee, "Workplace Flexibility and Communication Flows: A Structurational View", Journal of Applied Communication Research, 2017, 45(4), pp. 397-412.

[4] Faraj S. and S. Pachidi, "Beyond Uberization: The Coconstitution of Technology and Organizing", Organization Theory, 2021, 2(1), pp. 1-14.

[5] Leppälä M. and J.A. Espinosa, "Identifying Knowledge Brokers in Enterprise Social Media", Proceedings of the Annual Hawaii International Conference on System Sciences, 2020, pp. 481-490. doi: 10.24251/HICSS.2020.059
[6] Meyer, M. "The Rise of the Knowledge Broker", Science Communication, 2010, 32(1), pp. 118-127.

[7] Olejniczak, K., E. Raimondo, and T. Kupiec, "Evaluation Units as Knowledge Brokers: Testing and Calibrating an Innovative Framework", Evaluation, 2016, 22(2), pp. 168-189.

[8] Burt, R.S., "Structural Holes and Good Ideas", American Journal of Sociology, 2004, 110(2), pp. 349-399.

[9] Gitman, L.J., C. McDaniel, A. Shah, M. Reece, L. Koffel, B. Talsma, and J.C. Hyatt, Introduction to Business, OpenStax, 2018.

[10] Arena, M., J. Cross, J. Sims, and M. Uhl-Bien, "How to Catalyze Innovation in your Organization", MIT Sloan Management Review, 2017, 58(4), pp. 38-48.

[11] Schreyögg, G., and J. Sydow, "Organizing for Fluidity? Dilemmas of New Organizational Forms", Organization Science, 2010, 21(6), pp. 1251-1262.

[12] Leonardi, P.M., "Social Media, Knowledge Sharing, and Innovation: Toward a Theory of Communication Visibility", Information Systems Research, 2014, 25(4), pp. 796-816.

[13] Williams, T.A. and D.A. Shepherd, "Mixed Method Social Network Analysis Combining Inductive Concept Development, Content Analysis, and Secondary Data for Quantitative Analysis", Organizational Research Methods, 2017, 20(2), pp. 268-298.

[14] Munk, A.K., A.K. Madsen, and M. Jacomy, "Thinking through the Databody: Sprints as Experimental Situations", In A. Mäkitalo, T. Nicewonger, and M. Elam (Eds.), Designs for Experimentation and Inquiry: Approaching Learning and Knowing in Digital Transformation, 2019, Routledge, London, pp. 110-128.

[15] Venturini, T., A. Munk, and A. Meunier, "DataSprinting”, In C. Lury, R. Fensham, A. Heller-Nicholas, S. Lammes, A. Last, M. Michael, and E. Uprichard (Eds.), Routledge Handbook of Interdisciplinary Research Methods, Routledge, London, 2019, pp. 158163. doi: 10.4324/9781315714523-24

[16] Carrington, P.J., J. Scott, and S. Wasserman (Eds.), Models and Methods in Social Network Analysis (Vol. 28), Cambridge University Press, 2005.

[17] Huhtamäki, J., M.G. Russell, N. Rubens, and K. Still, "Ostinato: The Exploration-Automation Cycle of UserCentric, Process-Automated Data-Driven Visual Network Analytics", In S.A. Matei, M.G. Russell, and E. Bertino (Eds.), Transparency in Social Media, Springer, Cham, 2015, pp. 197-222.

[18] Freeman, L.C., "Visualizing Social Networks", Journal of Social Structure, 2000, 1(1), pp. 1-15.

[19] Leonardi, P.M., M. Huysman, and C. Steinfield, "Enterprise Social Media: Definition, History, and Prospects for the Study of Social Technologies in Organizations", Journal of Computer-Mediated Communication, 2013, 19(1), pp. 1-19.

[20] Kane, G.C., "Enterprise Social Media: Current Capabilities and Future Possibilities", MIS Quarterly Executive, 2015, 14(1), pp. 1-16.

[21] Treem, J.W. and P.M. Leonardi, "Social Media Use in Organizations: Exploring the Affordances of Visibility, Editability, Persistence, and Association”, Annals of the 
International Communication Association, 2013, 36(1), pp. 143-189.

[22] Finnish National Board on Research Integrity TENK, "The ethical principles of research with human participants and ethical review in the human sciences in Finland", 2019, https://tenk.fi/sites/default/files/202101/Ethical_review_in_human_sciences_2020.pdf.

[23] Knuth, D.E., "Literate Programming”, Computer Journal, 1984, 27(2), pp. 97-111. doi: 10.1093/comjnl/27.2.97

[24] Star, S.L. and J.R. Griesemer, "Institutional Ecology, 'Translations' and Boundary Objects: Amateurs and Professionals in Berkeley's Museum of Vertebrate Zoology, 1907-39", Social Studies of Science, 1989, 19(3), pp. 387-420. doi: 10.1177/030631289019003001
[25] Freeman, L.C., "A Set of Measures of Centrality Based on Betweenness", Sociometry, 1977, 40(1), pp. 35-41.

[26] Blondel, V.D., J.-L. Guillaume, R. Lambiotte, and E. Lefebvre, "Fast Unfolding of Communities in Large Networks", Journal of Statistical Mechanics: Theory and Experiment, 2008. doi: 10.1088/17425468/2008/10/P10008

[27] Bastian, M., S. Heymann, and M. Jacomy, "Gephi: An Open Source Software for Exploring and Manipulating Networks", Proceedings of the Third International AAAI Conference on Weblogs and Social Media, May 17-20, 2009, San Jose, California, USA, pp. 361-362. 